\title{
NLTE analysis of the solar potassium abundance (Research Note)
}

\author{
H.W. Zhang ${ }^{1,2}$, K. Butler ${ }^{2}$, T. Gehren ${ }^{2}$, J.R. Shi' ${ }^{2,3}$, and G. Zhao ${ }^{2,3}$ \\ 1 Department of Astronomy, School of Physics, Peking University, Beijing 100871, China \\ 2 Institut für Astronomie und Astrophysik der Universität München, Scheinerstrasse 1, 81679 München, Germany \\ e-mail: gehren@usm.lmu.de \\ ${ }^{3}$ National Astronomical Observatories, Chinese Academy of Sciences, Beijing 100012, China
}

Received 23 February 2006 / Accepted 31 March 2006

ABSTRACT

\begin{abstract}
We investigate the formation of neutral potassium lines in the solar photosphere based on extensive statistical equilibrium calculations to determine the solar potassium abundance. The computations are based on a 68-level potassium atomic model. Hydrogen collisions were supplied with an enhancement factor $S_{\mathrm{H}}=0.05$ to the classical Drawin formula. Synthetic line profiles calculated from the level populations according to the NLTE departure coefficients were compared with the observed solar spectral atlas. The solar potassium abundance based on theoretical model atmospheres is $\log \varepsilon_{\odot}(K)=5.12 \pm 0.03$, which agrees with the meteoritic value of $5.09 \pm 0.05$ compiled by Lodders.
\end{abstract}

Key words. line: formation - line: profiles - Sun: abundances

\section{Introduction}

The solar chemical abundance is one of the most important references in astronomy, so the description of our Galaxy's chemical evolution always begins with a detailed determination of the isotopic and elemental abundance distribution found in the solar system. Solar system abundances are determined from multiple sources where all have their own advantages and disadvantages (e.g. Grevesse \& Sauval 1998; Asplund et al. 2005; Sneden \& Lawler 2005). The majority of elements can be detected in pristine meteorites, allowing not only elemental but also isotopic compositions to be determined in direct laboratory analysis. Unfortunately, a very small subsample of meteorites does not show the effects of fractionation. Some elements are volatile due to their low condensation temperatures and hence are depleted in many meteorites (Lodders 2003).

The abundances of a very large number of elements can be determined by analyzing absorption lines found in the solar spectrum. The results rely on our knowledge of stellar atmospheric models and on the line formation process. Among the most important problems related to atmospheric line formation, there remains the assumption of local thermodynamic equilibrium (LTE), usually applied to the computation of atomic populations and radiative transfer (Gehren et al. 2001). For many ions the introduction of statistical equilibrium (NLTE) populations does not produce large changes with respect to LTE under solar conditions, due to atmospheric densities close to the main sequence that provide strong collisional interaction of all types of particles. However, depending on atomic structure and radiative interaction cross-sections, all ion populations deviate from LTE in the upper photospheric layers.

Together with the other one-electron systems, rubidium and cesium, neutral potassium has one of the smallest ionization energies in the periodic system. In the Sun and other near turnoff stars, its $\chi_{\text {ion }}=4.34$ is therefore a clear indicator for an extreme minority ion. Whereas $\mathrm{Na}$ I still constitutes $\sim 0.7 \%$ of the sodium atoms under conditions of the solar atmosphere, K I represents a minute $0.1 \%$, and it is well-known that such ions are particularly sensitive to deviations from LTE. This, however, depends on whether they are dominated by photoionization or collisions.

Such considerations have guided a number of analyses of the kinetic equilibrium of potassium in the past (e.g. Bruls et al. 1992; Takeda et al. 1996; Ivanova \& Shimansky 2000). Recent improvements in atomic data, methods, and computing capabilities make detailed line formation calculations of potassium ever more realistic.

In this paper we investigate the statistical equilibrium and formation of neutral potassium lines in the solar photosphere. The atomic model is presented in Sect. 2. In Sect. 3 the solar potassium abundance is determined from spectrum line-profile fits. The discussion fills the last section.

\section{Atomic model}

All calculations were carried out with a revised version of the DETAIL program (Butler \& Giddings 1985) using accelerated lambda iteration (see Gehren et al. 2001, 2004 for details).

Potassium has a relatively simple term structure, which is listed in the NIST data bank (see also Moore 1971) up to $L=3$. Some more ${ }^{2} \mathrm{G}$ terms were added from our own calculations. As seen in Fig. 1, the ${ }^{2} \mathrm{~S}$ to ${ }^{2} \mathrm{G}$ terms of our atomic model are complete up to $n=10$, roughly $3 \%$ below the ionization energy. Whenever available, additional terms were added up to $n=20$. Since only the resonance and first subordinate lines are strong enough to carry substantial radiative flux, fine structure was neglected except for the $4 p$ term. The total number of levels is 67 plus the ground state of K II. No attempt was made to include terms with higher angular momentum $L>4$ since their influence was found negligible in an earlier investigation of the 


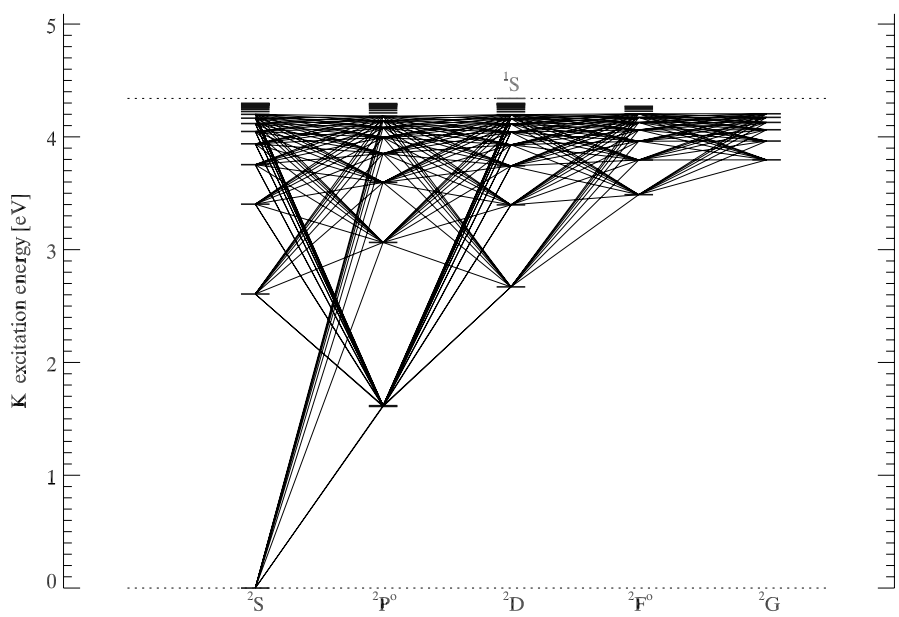

Fig. 1. Adopted level structure of the KI atom taken mostly from the NIST data bank. Explicitly calculated line transitions are indicated.

similar Na I (Baumüller et al. 1998). Quite generally, such highly excited levels lead to transitions in the millimeter and the radio wavelength domain, where cool star spectra no longer emerge from the quiet photosphere.

Oscillator strengths and photoionization cross-sections are from Butler (2000). They refer to a 9-state R-matrix calculation carried out using the Opacity Project (Berrington et al. 1987) codes as provided by Badnell ${ }^{1}$. The data are available from Butler $^{2}$ by request. The photoionization cross-sections are different from hydrogenic approximations as is shown in Fig. 2. While the ground state, $4 \mathrm{~s}^{2} \mathrm{~S}$, shows a very small photoionization cross-section near the threshold, even compared with a hydrogenic approximation, the cross-section of the first excited level, $4 \mathrm{p}^{2} \mathrm{P}^{\mathrm{o}}$, is roughly ten times larger than provided by Kramers' formula. Radiative transitions in the NLTE model calculations were therefore all treated with calculated crosssections. As usual, background opacities were calculated with an opacity sampling code based on the line lists made available by Kurucz (1992). Since background opacities affect the photoionization rates directly, their consideration is important (see e.g. Collet et al. 2005).

Both electron and hydrogen collisions were applied using the approximations of van Regemorter (1962) and Drawin (1968, 1969), respectively, for bound-bound electron and hydrogen collisions for the allowed transitions. Hydrogen collisions were supplied with an enhancement factor $S_{\mathrm{H}}=0.05$ to the classical Drawin (1968) formula, for which results are given below. Forbidden transitions were approximated using a collision strength of $\Omega=1$, together with Allen's (1973) formula. The calculation of bound-free electron collisions followed the approximation of Seaton (1962) with appropriate Gaunt factors. Similar to the Na I problem, hydrogen collisions with $\mathrm{KI}$ atoms do not change the populations within a broad range of parameters, i.e. $0<S_{\mathrm{H}}<0.1$. Thermalization of the populations by hydrogen collisions starts to become important for $S_{\mathrm{H}}>1$.

The departure coefficients $b_{k}=n_{k}^{\mathrm{NLTE}} / n_{k}^{\mathrm{LTE}}$ in the solar atmosphere are presented in Fig. 3. The results are best characterized by the overpopulation of ground state $4 s^{2} S_{1 / 2}$ and the first excited level $4 \mathrm{p}^{2} \mathrm{P}^{\mathrm{o}}$ in the higher atmospheric layers beyond $\log \tau=-0.5$. The departure coefficients diverge outside at $\operatorname{low} \log \tau$, while the excited states follow the

\footnotetext{
${ }^{1}$ http://www. amddp.phys.strath.ac.uk/UK_RmaX

2 butler@usm.lmu.de
}

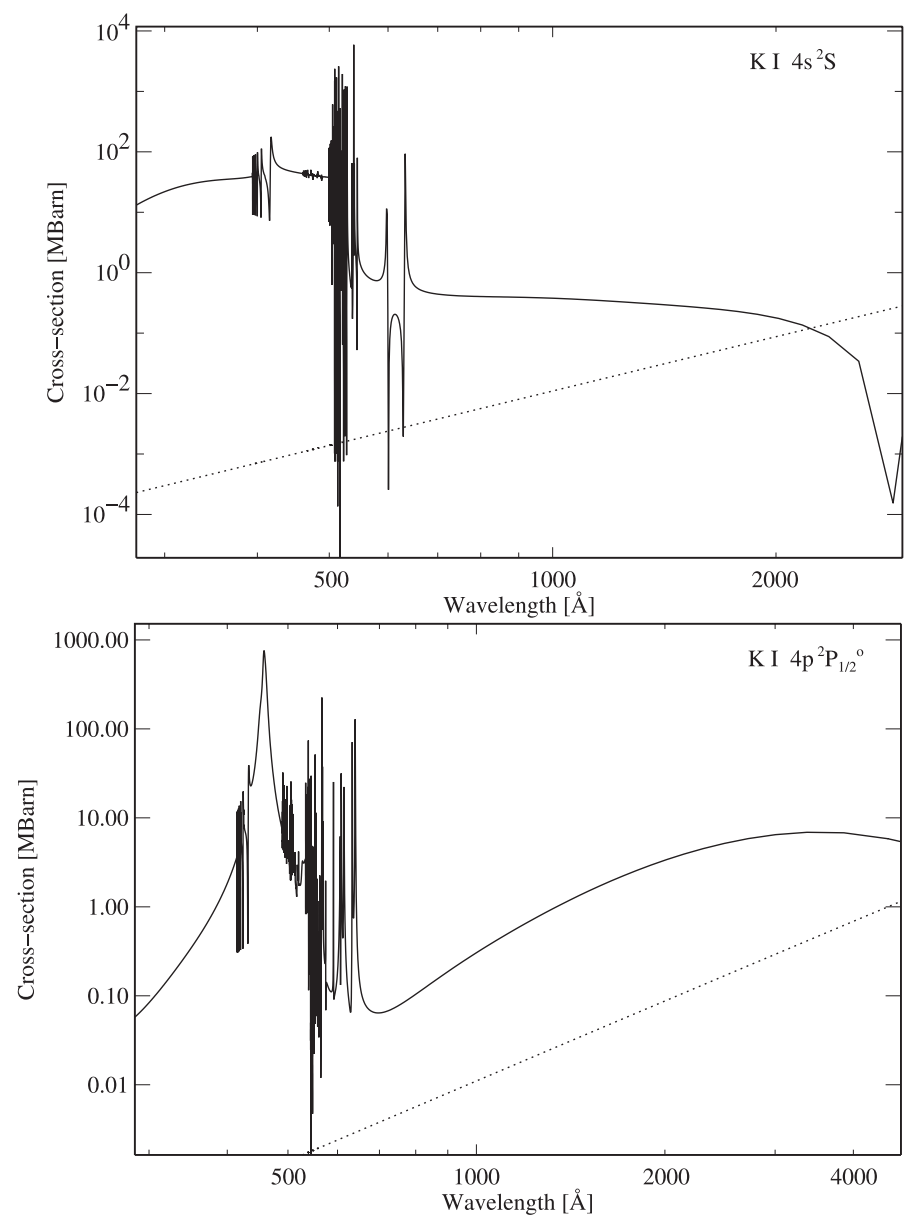

Fig. 2. Typical examples of photoionization cross-sections (in MBarn), calculated for the ground state (top) and the first excited level (bottom) of K I. The hydrogenic approximation is Kramers' (1923) formula, represented by the dotted line.

overpopulation to a lesser extent and only in deeper atmospheric layers, which is very similar to what is found for $\mathrm{Na}$ I (e.g. Baumüller et al. 1998). This is not unexpected since both potassium and sodium atomic systems have similar energy level structures and are dominated by collisions. Potassium lines therefore tend to be stronger in an NLTE environment than under LTE, so most of the LTE abundance determinations require negative corrections.

\section{Analysis of $\mathrm{K} \mathrm{I}$ lines in the solar spectrum}

In our solar analysis we investigated the formation of K I lines in various solar model atmospheres, which are all plane-parallel and in hydrostatic equilibrium, with convective energy transfer calculated from the mixing-length theory. Abundances are based on line-profile fitting.

\subsection{Solar atmospheric models}

Most of the models are typical line-blanketted models making use of either Kurucz's (1992) opacity distribution functions (ODF) or of opacity sampling (OS) methods. Since the results differ by less than 0.03 in mean NLTE abundances, we concentrated on our own ODF model calculated with opacities rescaled to a solar iron abundance of $\log \varepsilon_{\odot}(\mathrm{Fe})=7.51$. This value was originally derived from Anders \& Grevesse (1989). More details are found in Gehren et al. (2001). The solar parameters of 

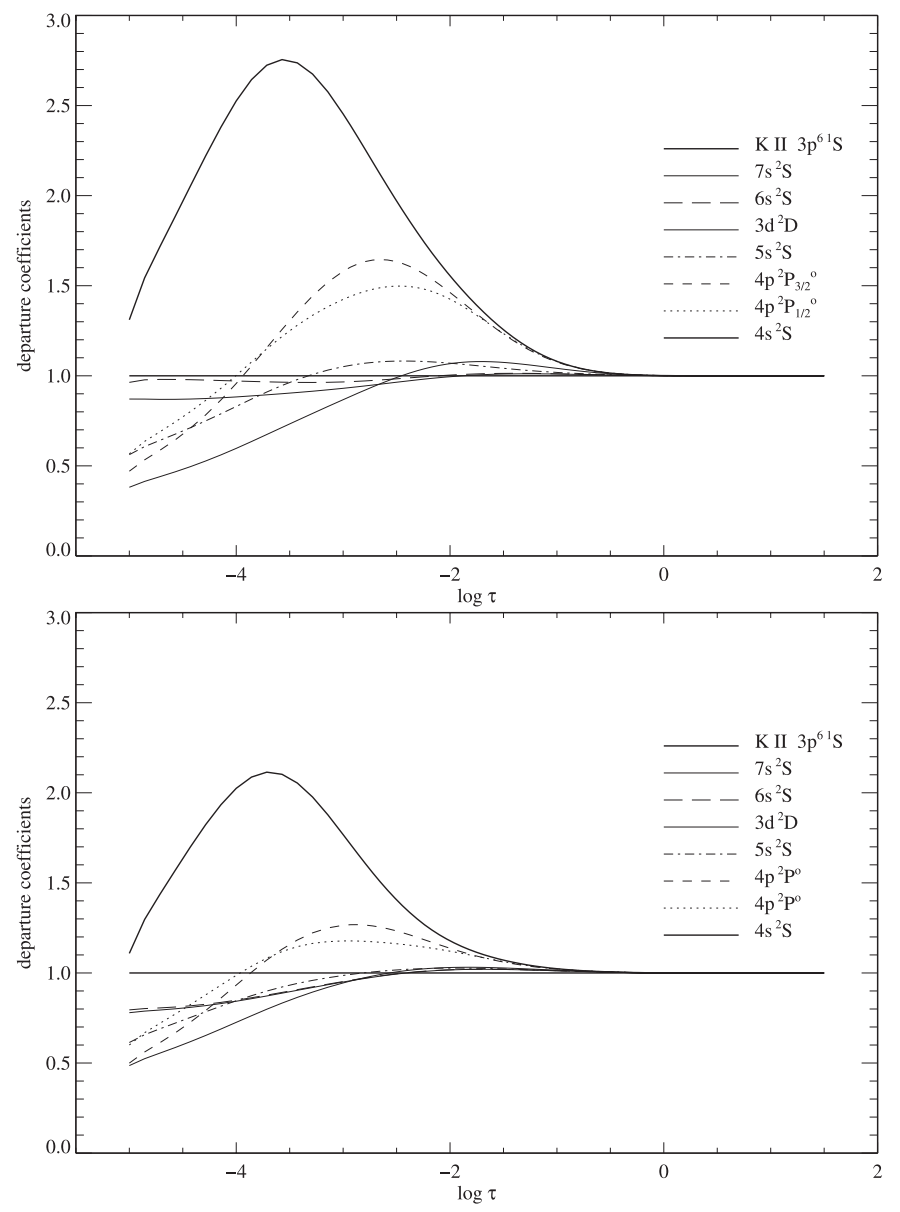

Fig. 3. Departure coefficients $b_{n}$ for some levels of $\mathrm{K} \mathrm{I}$ in the ODF solar atmospheric model. The kinetic equilibrium calculations are described in the text. Top: $S_{\mathrm{H}}=0.05 ;$ bottom: $S_{\mathrm{H}}=1.0$.

$T_{\text {eff }}=5780 \mathrm{~K}, \log g=4.44,[\mathrm{Fe} / \mathrm{H}]=0.00, \xi_{\mathrm{t}}=0.90 \mathrm{~km} \mathrm{~s}^{-1}$ were adopted for all calculations of theoretical models.

The second set of models is represented by the empirical solar models of Holweger \& Müller (1974, HM74) and Maltby et al. (1986). Again, the mean abundances are within 0.03 dex, though slightly different from the abundances found from theoretical models, so the HM74 model was used for comparison.

\subsection{Atomic line data}

Seven observable lines in the wavelength interval $5000 \AA<\lambda<$ $13000 \AA$ were used in our analysis, although the resonance line at $7664.92 \AA$ is badly blended by a telluric $\mathrm{O}_{2}$ line in the solar spectrum.

To determine the solar abundance it is necessary to know the oscillator strengths ( $g f$ values) of the spectral lines. We used the $g f$ values calculated by Butler (2000). Collisional line broadening through the van der Waals interaction with hydrogen atoms is important only for the relatively strong $\mathrm{K}$ I resonance lines. The data used here are based on the Anstee \& O'Mara (1991, 1995) tables, with the exception of the resonance lines. Their values were increased by $\Delta \log C_{6}=0.11$ dex to fit the solar line wings. The corresponding line data for our line fits are reproduced in Table 1.
Table 1. Atomic data and abundance results for K I lines. Solar K I abundances in the last column refer to NLTE calculations using our standard ODF model atmosphere. A colon marks the result for the telluric blend.

\begin{tabular}{rrcrrc}
\hline \hline$\lambda[\AA]$ & $E[\mathrm{eV}]$ & Transition & $\log g f$ & $\log C_{6}$ & $\log \varepsilon_{\odot}$ \\
\hline 5801.75 & 1.617 & $4 \mathrm{p}^{2} \mathrm{P}_{3 / 2}^{\mathrm{o}}-7 \mathrm{~s}^{2} \mathrm{~S}_{1 / 2}$ & -1.605 & -31.20 & 5.15 \\
6938.77 & 1.617 & $4 \mathrm{p}^{2} \mathrm{P}_{3 / 2}^{\mathrm{o}}-6 \mathrm{~s}^{2} \mathrm{~S}_{1 / 2}$ & -1.119 & -30.27 & 5.06 \\
7664.92 & 0.000 & $4 \mathrm{~s}^{2} \mathrm{~S}_{1 / 2}-4 \mathrm{p}^{2} \mathrm{P}_{3 / 2}^{\mathrm{o}}$ & 0.134 & -31.00 & $5.14:$ \\
7698.98 & 0.000 & $4 \mathrm{~s}^{2} \mathrm{~S}_{1 / 2}-4 \mathrm{p}^{2} \mathrm{P}_{1 / 2}^{\mathrm{o}}$ & -0.169 & -31.00 & 5.14 \\
11769.63 & 1.617 & $4 \mathrm{p}^{2} \mathrm{P}_{3 / 2}^{\mathrm{o}}-3 \mathrm{~d}^{2} \mathrm{D}_{3 / 2}$ & -0.452 & -30.80 & 5.10 \\
12432.23 & 1.610 & $4 \mathrm{p}^{2} \mathrm{P}_{1 / 2}^{\mathrm{o}}-5 \mathrm{~s}^{2} \mathrm{~S}_{1 / 2}$ & -0.460 & -30.08 & 5.10 \\
12522.10 & 1.617 & $4 \mathrm{p}^{2} \mathrm{P}_{3 / 2}^{\mathrm{o}}-5 \mathrm{~s}^{2} \mathrm{~S}_{1 / 2}$ & -0.150 & -30.08 & 5.14 \\
\hline
\end{tabular}

\subsection{Line-profile fitting}

The observed solar flux spectrum was taken from the Kitt Peak Atlas (Kurucz et al. 1984). The spectral line-profile fitting procedure made use of the interactive spectrum synthesis program, SIU, which is a IDL/Fortran-based software package (Reetz 1990).

Before discussing the individual lines it is necessary to recall that NLTE line-formation results depend on the choice of the hydrogen collision enhancement factor $S_{\mathrm{H}}$. Our NLTE test calculations cover a wide range in the scale factor from $S_{\mathrm{H}}=$ 0 to 10 . The full analysis of all selected potassium lines allows the choice of a reasonable value for $S_{\mathrm{H}}=0.05$. In fact, as noted above, the results are not very sensitive to hydrogen collisions below $S_{\mathrm{H}}=0.1$. They start to become more thermalized only above $S_{\mathrm{H}}=0.5$ (see Fig. 3), and for such values the resonance line profile at $7698 \AA$ no longer fits the solar spectrum.

The comparison of synthetic profiles calculated from the level populations according to the NLTE departure coefficients with the observed profiles for all selected potassium lines, except for the resonance line at $7664.92 \AA$ (see below), is shown in Fig. 4. In order to match the observed spectral lines, the synthetic spectra were convolved with a mean solar rotational velocity of $1.8 \mathrm{~km} \mathrm{~s}^{-1}$, and a radial-tangential macroturbulence $\Xi_{\mathrm{rt}}$ that is found to vary for lines of different mean formation depth between 1.5 and $5.0 \mathrm{~km} \mathrm{~s}^{-1}$. For the weak line at $5801 \AA$ and for the near infrared lines at 12432 and $12522 \AA$, the observed line profiles depend to some extent on the choice of the continuum reference level. Accepting the KPNO solar atlas normalization would mean that the continuum level is never reached within $\pm 10 \AA$. Our local continuum thus implies a depression of $\sim 0.02, \sim 0.01$ and $\sim 0.013$ of the original flux normalization, respectively. The figures show that our synthetic profiles based on the NLTE calculations fit the observational solar spectrum very well, whereas the LTE profile of the $7698 \AA$ line is generally too weak in the line core. The $6938 \AA$ line can only be reproduced with a low potassium abundance. This can be explained neither by a bad continuum placement nor as the result of inaccurate NLTE populations, because $\lambda \lambda$ 5801, 6938, and $12522 \AA$ all have the same lower level. Presumably there exists a problem with the oscillator strength of this line.

The profile of the other resonance line at $7664 \AA$ was also calculated in order to check its compatibility with the $7698 \AA$ line. The resulting blend was based on the profile of the adjacent $\mathrm{O}_{2}$ line near $7665.9 \AA$. The undisturbed red wing of that telluric line was used to extract a saturated symmetric profile. This profile was copied to the position of the other $\mathrm{O}_{2}$ line at $7664.8 \AA$, and the blend of solar $\mathrm{KI}$ and the telluric $\mathrm{O}_{2}$ lines was fitted using the double $\mathrm{O}_{2}$ line as an additional absorber. Figure 5 gives 

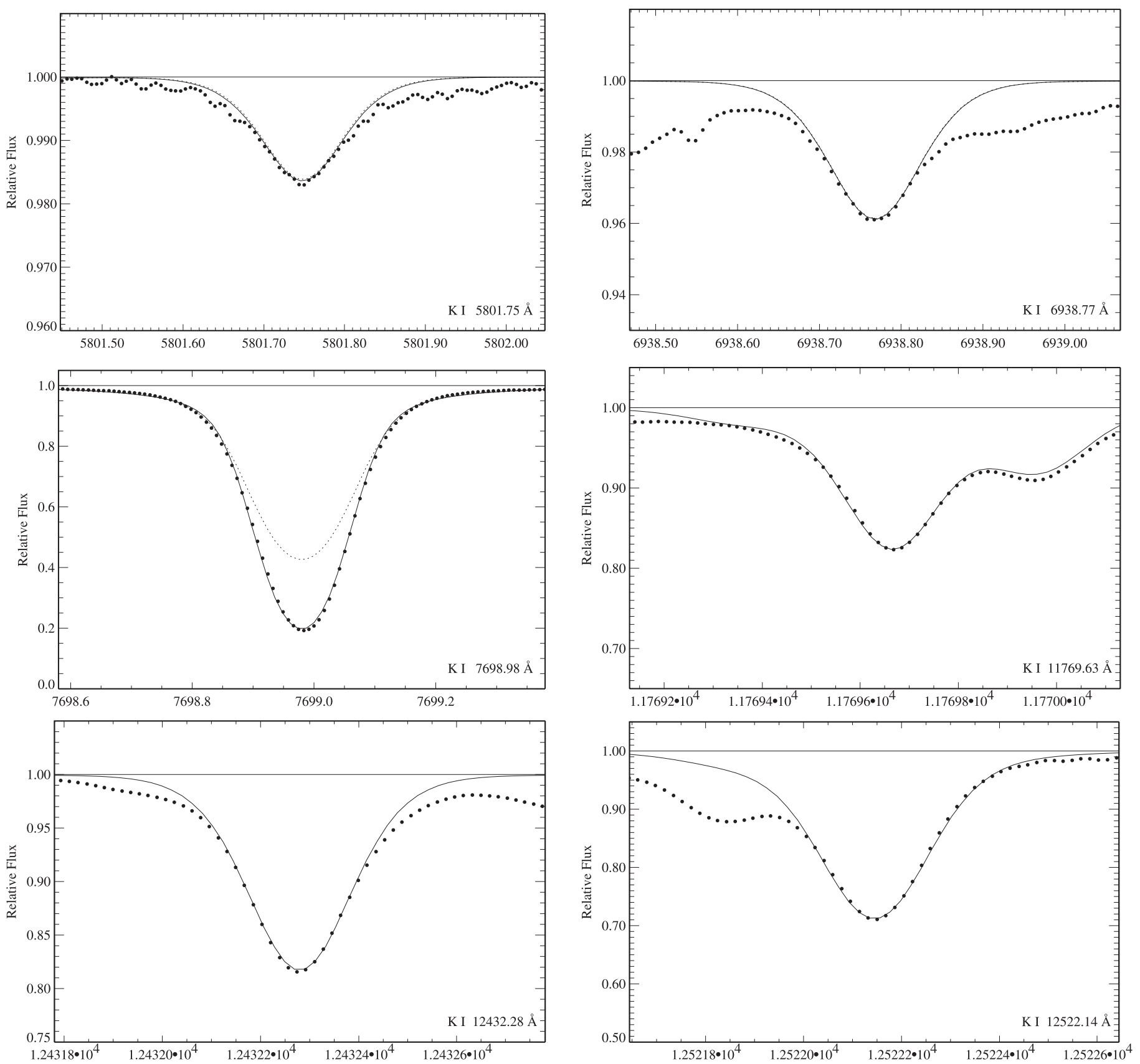

Fig. 4. Synthetic NLTE flux profiles of selected K I lines compared with the observed solar spectrum of Kurucz et al. (1984). Solar continuum fluxes were re-normalized for the lines at $12432 \AA(-1.7 \%)$ and $12522 \AA(-1.3 \%)$. Continuous profiles are NLTE calculations calculated with the departure coefficients of Fig. 3; dotted are LTE profiles using different abundances for comparison.

an impression of the good fit to the solar spectrum, where the K I line is calculated with the same abundance and macroturbulence velocity as the other resonance line.

\section{Discussion}

The potassium abundance is a free parameter in our line-profile fitting procedures. Table 1 presents individual abundances obtained from our final line fits. The synthetic profiles were all calculated from NLTE populations based on a potassium abundance of $\log \varepsilon_{\odot, \mathrm{K}}=5.05$. However, it turns out that the NLTE populations are not sensitive to the specification of the solar potassium abundance. Changing the solar $\mathrm{K}$ abundance by 0.07 dex produces departure coefficients that in turn change the line abundance by less than 0.005 dex. Our NLTE analysis of the potassium lines in the solar spectrum brings the resonance line abundance into agreement with the mean abundance derived from all the subordinate lines, i.e. the dispersion of our potassium abundance that results from different lines is small. The mean solar potassium abundance of all six lines excluding $7664.92 \AA$ is $\log \varepsilon_{\odot, \mathrm{K}}=5.115 \pm 0.034$, which is in perfect agreement with the meteoritic abundance value of 5.12 presented in the compilation of Grevesse \& Sauval (1998) and still in reasonable agreement with the more recent value of $5.09 \pm 0.05$ given by Lodders (2003) or $5.08 \pm 0.07$ as cited by Asplund et al. (2005).

Besides the theoretical solar ODF model, the semi-empirical model of Holweger \& Müller (HM, 1974) is another widely used solar model. The most important difference between the two model types with respect to line formation is the temperature 


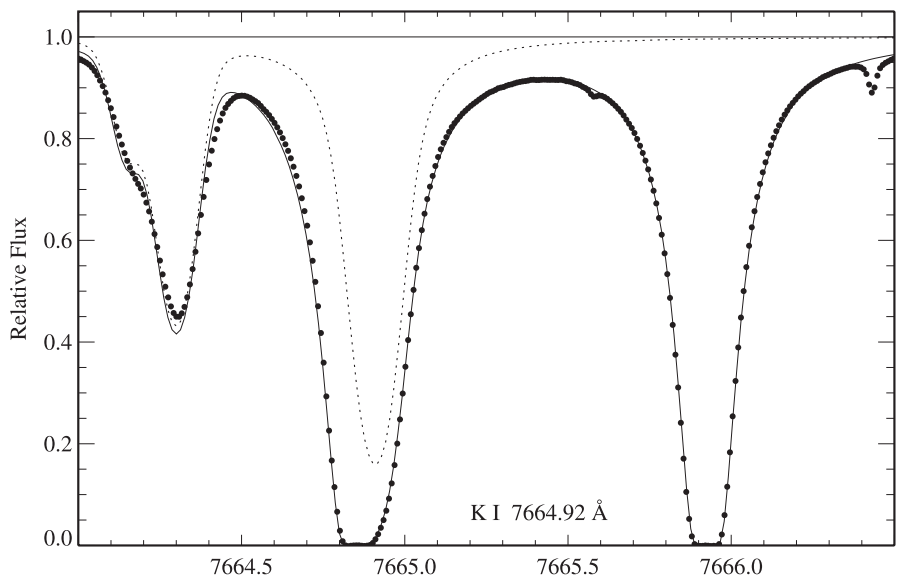

Fig. 5. Synthetic flux profile of KI Line at $7664.92 \AA$ calculated with the departure coefficients of Fig. 3 compared with the observed solar spectrum of Kurucz et al. (1984, filled circles). The blend with the telluric $\mathrm{O}_{2}$ lines is described in the text. The dotted curve represents the synthetic spectrum, including the $\mathrm{K} I$ line, and the continuous curve shows the same spectrum modified by the $\mathrm{O}_{2}$ lines.

Table 2. NLTE corrections to the solar K I abundances using our standard ODF model atmosphere. $\Delta F_{\mathrm{c}}$ refers to the change of the continuum relative to the original normalization in the KPNO atlas. Equivalent widths are measured using the final NLTE flux profile fits (meant to care for faint unknown blends). The resulting abundance differences between NLTE and LTE populations are calculated for the equivalent widths, because some profiles cannot be fitted with LTE populations.

\begin{tabular}{rrrcl}
\hline \hline$\lambda[\AA]$ & $\Delta F_{\mathrm{c}}$ & $W_{\lambda}$ & $\Delta \log \varepsilon_{\odot, \mathrm{K}}$ & Remarks \\
\hline 5801.75 & -0.016 & 2.2 & -0.06 & Terrestrial blends \\
6938.77 & 0.000 & 4.9 & -0.07 & Uncertain continuum \\
7698.98 & 0.000 & 169.9 & -0.29 & \\
11769.63 & -0.005 & 49.1 & -0.13 & $\mathrm{CN}$ blend \\
12432.23 & -0.017 & 51.8 & -0.15 & \\
12522.10 & -0.022 & 84.6 & -0.22 & $\mathrm{C}_{2}$ blend \\
\hline
\end{tabular}

stratification (see Gehren et al. 2001, for more detail). We also derive the potassium abundance based on the HM74 model, and the result is $\log \varepsilon_{\odot, \mathrm{K}}=5.207 \pm 0.044$, i.e. slightly higher than the ODF result, and systematically above the meteoritic abundance.

Lambert \& Luck (1978) derived the solar potassium abundance from an LTE analysis of five weak and medium-strong lines and their solar potassium abundance was $\log \varepsilon_{\odot, \mathrm{K}}=5.14$. This low mean value results from a fit to the significantly blended blue line at $4044.2 \AA$. A mean abundance of $\log \varepsilon_{\odot, \mathrm{K}}=$ $5.19 \pm 0.08$ is obtained by restricting the analysis to the other four lines in common with our line sample.

Repeating their LTE analysis for the four lines results in $\log \varepsilon_{\odot, \mathrm{K}}=5.30 \pm 0.05$ for our HM74 model using solar $f l u x$ spectra. Both microturbulence and damping constants are not important for these lines. The difference between our calculations are probably due to the following reasons:

- the difference between our $g f$-values for the $5801 \AA$ line is 0.09 dex;

- the measurement of their equivalent widths seems to result in systematically lower values; and

- what is generally called the Holweger-Müller solar model may be different in that our HM74 model refers to the temperature stratification but has its partial pressures adjusted to the abundances of Anders \& Grevesse (1989).
Finally there could remain a small systematic difference in abundance determinations from solar intensity and flux spectra. Takeda et al. (1996) performed NLTE calculations of potassium. They found that the potassium abundance derived from $7699 \AA$ resonance line is significantly affected by NLTE effects and obtained $\log \varepsilon_{\odot, \mathrm{K}} \sim 5.1$ as the solar potassium abundance, in good agreement with our result.

The NLTE computations of Ivanova \& Shimansky (2000) are based on a 36-level model of the neutral potassium atom. From comparison with the KPNO solar atlas (Kurucz et al. 1984) and using the ODF solar model of Kurucz (1992), they obtain a NLTE solar potassium abundance of $\log \varepsilon_{\odot, \mathrm{K}}=5.14$ using the profiles and equivalent widths of four lines including the resonance line at $7698 \AA$. While their departure coefficients are apparently very similar to ours, their line fits differ significantly. The low value of their resonance line abundance determines the mean value. It refers to a mean microturbulence value of $\xi=1.2 \mathrm{~km} \mathrm{~s}^{-1}$. Such a high isotropic microturbulence is in fact adequate when analyzing some of the Fe II lines in the solar spectrum, but it is far too high for the $\mathrm{K}$ I resonance line. This is documented in their Fig. 3, where the corresponding line profile is too broad. Fitting that observed profile with the usual value of $0.9 \mathrm{~km} \mathrm{~s}^{-1}$ leads to an 0.08 dex increase in the required line abundance.

The static solar atmospheric potassium abundance seems to be well determined by NLTE calculations and in sufficient agreement with the meteoritic value. Remaining fine tuning, however, requires the consideration of hydrodynamical atmospheric models (Asplund et al. 2005).

Acknowledgements. This project was supported by the Deutsche Forschungsgemeinschaft (DFG) under grants Ge490/26-1 and 446 CHV 112 and by the National Natural Science Foundation of China under grants No. 10433010 and 10521001. ZHW and SJR thank the Institute of Astronomy and Astrophysics of Munich University for the warm hospitality during a productive stay in 2004.

\section{References}

Allen, C. W. 1973, Astrophysical Quantities, 3rd edn. (London: Athlone Press) Anders, E., \& Grevesse, N. 1989, Geochim. Cosmochim. Acta, 53, 197 Anstee S. D., \& O'Mara, B.J. 1991, MNRAS, 253, 549 Anstee S. D., \& O'Mara, B.J. 1995, MNRAS, 276, 859

Asplund M., Grevesse N., \& Sauval, A. J. 2005, ASP Conf. Ser., 336, 25 Baumüller, D., Butler, K., \& Gehren, T. 1998, A\&A, 338, 637 Berrington, K. A., Burke, P. G., Butler, K., et al. 1987, J. Phys. B, 20, 6379

Bruls, J. H., Rutten, R. J., \& Shchukina 1992, A\&A, 265, 237

Butler, K. 2000, unpublished

Butler, K., \& Giddings J. 1985, Newsletter on the analysis of astronomical spectra No. 9, University of London

Collet, R., Asplund, M., \& Thévenin, F. 2005, A\&A, 442, 643

Drawin, H. W. 1968, Z. Phys., 211, 404

Drawin, H. W. 1969, Z. Phys., 225, 483

Gehren, T., Butler, K., Mashonkina, L., et al. 2001, A\&A, 366, 981

Gehren, T., Liang, Y. C., Shi J. R., et al. 2004, A\&A, 413, 1045

Grevesse, N., \& Sauval, A. J. 1998, Space Sci. Rev., 85, 161

Holweger, H., \& Müller, E. A. 1974, Sol. Phys., 39, 19

Ivanova, D. V., \& Shimansky, V. V. 2000, Astron. Rep., 44, 376

Kramers, H. A. 1923, Phil. Mag., 46, 836

Lambert, D. L., \& Luck, R. E. 1978, MNRAS, 183, 79

Lodders, K. 2003, ApJ, 591, 1220

Kurucz, R. L. 1992, Rev. Mex. Astron. Astrof., 23, 45

Kurucz, R. L., Furenlid, I., Brault, J., \& Testerman, L. 1984, Solar Flux Atlas from 296 to $1300 \mathrm{~nm}$, Kitt Peak National Solar Observatory

Maltby, P., Avrett, E. H., Carlsson, M., et al. 1986, ApJ, 306, 284

Moore, C. E. 1971, Atomic Energy Levels Vol. I, NSDRS-NBS35, US Government Printing Office, Washington DC

Reetz, J. K. 1990, unpublished

Seaton, M. J. 1962, in Atomic and Molecular Processes (New York: Acad. Press)

Sneden, C., \& Lawler, J. E. 2005, ASP Conf. Ser., 336, 11

Takeda, Y., Kato, K., Watanabe Y., et al. 1996, PASJ, 48, 511

van Regemorter, H. 1962, ApJ, 136, 906 